\title{
ON A UNIQUE CONTINUATION PROPERTY RELATED TO THE BOUNDARY STABILIZATION OF MAGNETOHYDRODYNAMIC EQUATIONS*
}

BY

\author{
CĂTĂLIN-GEORGE LEFTER
}

\begin{abstract}
We prove a unique continuation result for a system of Stokes type equations in space dimension 2. This result is useful in the study of boundary stabilization of magnetohydrodinamic equations with boundary controllers.

Mathematics Subject Classification 2000: 93D15, 35Q35, 76W05, 35Q30, 35Q60, 93B07.

Key words: unique continuation, magnetohydrodynamic equations, feedback stabilization, stream function.
\end{abstract}

1. Introduction. This paper is concerned with a unique continuation result useful in the study of the local stabilization for the magnetohydrodynamic (MHD) equations in space dimension 2, with boundary controllers. The usual procedure for reducing a boundary controlled system to a system with controllers distributed in a subdomain is to extend the initial domain to a slightly larger domain, consider then the system in this new domain and control it with a controller distributed in the extension. The boundary controller for the former problem is the trace of the solution to the latter problem.

For the stabilization of the MHD equations with feedback controllers distributed in subdomains, we mention the paper [13] in space dimension 3 and [14] for the 2 dimensional case. However, these results are not directly applicable following the above procedure. The special feature of the MHD

\footnotetext{
${ }^{*}$ This work was supported by the CNCSIS grant PN II ID 404/2007-2010.
} 
system is that the second equation, verified by the magnetic field, needs to have a divergence free right hand side. By using localized controllers, after applying the Leray projector, the controller becomes, usually, distributed in the whole domain and the above reduction to the internal controllers case fails to work. That is why a special form of the internal controller for the second extended equation must be used; this yields a particular unique continuation property, which is proved in the paper, in the case of simply connected two dimensional domains.

The method for stabilization, which is used in the above mentioned papers, is to linearize the system around the stationary state and then construct a feedback controller stabilizing the linear system. Then, it is shown that the same controller stabilizes, locally in a specified space, the nonlinear system. The stabilization of the linearized system is obtained via a spectral decomposition of the elliptic part. The linear system is projected on the stable and unstable subspaces corresponding to this decomposition. The unstable subspace is finite dimensional and the corresponding projected system is exactly controllable, as a consequence of the approximate controllability of the original linearized system. One constructs thus a feedback stabilizing this finite dimensional linear system. The projected system on the stable subspace is asymptotically stable and the feedback for the finite dimensional system is stabilizing the initial linearized equations. The key point in all this methodology of local stabilization is the approximate controllability of the linearized system and this is equivalent to a unique continuation property for the adjoint system.

For the method of spectral decomposition applied to Navier-Stokes equations with with internal controllers we mention the papers of BARBU [2] and BARbu and Triggiani [6]. Their approach for the stabilization of the finite dimensional projected equation relies on a Kalman type condition which is verified using a unique continuation property for a stationary Stokes type system. In [4] and [5], BARBU, LASIECKA and TRIGGIANI treated the stabilization of Navier-Stokes equations with boundary tangential controllers. We mention also the references [12],[15],[16] for the linearization method in the study of hydrodynamical stability and the existence of invariant manifolds.

2. Preliminaries and main result. Let $\Omega \subset \mathbb{R}^{2}$ be a bounded, simply connected open set with $C^{2}$ boundary $\partial \Omega$ and let $\omega \subset \subset \Omega$ an open subset of $\Omega$. Let $T>0$ and $Q=\Omega \times(0, T), \Sigma=\partial \Omega \times(0, T), \mathbf{n}$ is the unit 
exterior normal to $\partial \Omega, Q_{\omega}=\omega \times(0, T)$.

We consider the following MHD controlled system:

(1)

$$
\begin{cases}\frac{\partial y}{\partial t}-\nu \Delta y+(y \cdot \nabla) y-(B \cdot \nabla) B+\nabla\left(\frac{1}{2} B^{2}+p\right)=f+\chi_{\omega} u & \text { in } Q \\ \frac{\partial B}{\partial t}+\eta \operatorname{curl} \operatorname{curl} B+(y \cdot \nabla) B-(B \cdot \nabla) y=\chi_{\omega} P_{\omega} v & \text { in } Q \\ \nabla \cdot y=0, \nabla \cdot B=0 & \text { in } Q \\ y=0, B \cdot \mathbf{n}=0, \operatorname{rot} B=0 & \text { on } \Sigma \\ y(\cdot, 0)=y_{0}, B(\cdot, 0)=B_{0} & \text { in } \Omega\end{cases}
$$

The functions that appear in the system have the following physical meaning: $y=\left(y_{1}, y_{2}\right)^{T}: \Omega \times(0, T) \rightarrow \mathbb{R}^{2}$ is the velocity field, $p: \Omega \times(0, T) \rightarrow \mathbb{R}$ is the pressure, $B=\left(B_{1}, B_{2}\right)^{T}: \Omega \times(0, T) \rightarrow \mathbb{R}^{2}$ is the magnetic field and $f=\left(f_{1}, f_{2}\right)^{T}: \Omega \rightarrow \mathbb{R}^{2}$ represents the density of the exterior forces $\left((\cdots)^{T}\right.$ denotes the matrix transpose). The coefficients $\nu, \eta$ are the positive kinematic viscosity and the magnetic resistivity coefficients. We denote by

$$
\operatorname{rot} B=\frac{\partial B_{2}}{\partial x_{1}}-\frac{\partial B_{1}}{\partial x_{2}}
$$

the scalar version of the curl operator. We also note the formula curl curl $B$ $=-\Delta B+\nabla \operatorname{div} B$. So, knowing the fact that the solution $B$ will remain divergence free, we may write $-\Delta B$ instead of curl curl $B$ but we kept the notation in order to emphasize that the system models in fact phenomena in a 3 dimensional cylindrical body and the data depend only on $x_{1}, x_{2}$ variables.

The functions $u, v: \omega \times(0, T) \rightarrow \mathbb{R}^{2}, u, v \in \mathcal{U}:=L^{2}\left(0, T ;\left(L^{2}(\omega)\right)^{2}\right)$ are the controllers and $\chi_{\omega}: L^{2}(\omega) \rightarrow L^{2}(\Omega)$ is the operator extending the functions in $L^{2}(\omega)$ with 0 to the whole $\Omega$. We denote by $P_{\omega}, P_{\Omega}$ the Leray projectors corresponding to the domains $\omega, \Omega$.

In order to write (1) in an abstract form we define the following two operators:

$$
\begin{gathered}
A_{1} y=-P_{\Omega} \Delta y \text { for } y \in D\left(A_{1}\right) \\
A_{2} B=\operatorname{curl}(\operatorname{curl} B) \text { for } B \in D\left(A_{2}\right)
\end{gathered}
$$

where, denoting by

$$
H=\left\{y \in\left(L^{2}(\Omega)\right)^{2}: \operatorname{div} y=0, y \cdot \mathbf{n}=0 \text { on } \partial \Omega\right\},
$$




$$
V_{1}=H \cap\left(H_{0}^{1}(\Omega)\right)^{2}, V_{2}=H \cap\left(H^{1}(\Omega)\right)^{2},
$$

we have

$$
\begin{gathered}
D\left(A_{1}\right):=\left(H^{2}(\Omega)\right)^{2} \cap V_{1}, \\
D\left(A_{2}\right):=\left\{B \in\left(H^{2}(\Omega)\right)^{2} \cap V_{2} \mid \operatorname{rot} B=0 \text { on } \partial \Omega\right\} .
\end{gathered}
$$

With no loss of generality we will suppose that $\nu=\eta=1$ and system (1) may thus be written as

$$
\left\{\begin{array}{l}
y^{\prime}+A_{1} y+P_{\Omega}(y \cdot \nabla) y-P_{\Omega}(B \cdot \nabla) B=P_{\Omega} f+P_{\Omega}\left(\chi_{\omega} u\right) \\
B^{\prime}+A_{2} B+(y \cdot \nabla) B-(B \cdot \nabla) y=P_{\Omega} \chi_{\omega} P_{\omega} v=\chi_{\omega} P_{\omega} v \\
y(0)=y_{0}, B(0)=B_{0}
\end{array}\right.
$$

Consider for a given $f \in\left(H^{-1}(\Omega)\right)^{2}$ a steady state variational solution $(\bar{y}, \bar{B}, \bar{p}) \in V_{1} \times V_{2} \times L^{2}(\Omega)$ of $(1):$

$$
\begin{cases}-\Delta \bar{y}+(\bar{y} \cdot \nabla) \bar{y}+\nabla\left(\frac{1}{2} \bar{B}^{2}\right)-(\bar{B} \cdot \nabla) \bar{B}+\nabla \bar{p}=f & \text { in } \Omega, \\ \operatorname{curl} \operatorname{curl} \bar{B}+(\bar{y} \cdot \nabla) \bar{B}-(\bar{B} \cdot \nabla) \bar{y}=0 & \text { in } \Omega, \\ \nabla \cdot \bar{y}=0, \nabla \cdot \bar{B}=0 & \text { in } \Omega, \\ \bar{y}=0, \bar{B} \cdot \mathbf{n}=0, \operatorname{rot} \bar{B}=0 & \text { on } \Sigma .\end{cases}
$$

We assume the following regularity assumption for our stationary solution:

(H) $\quad \bar{y}, \bar{B} \in W^{2, \infty}(\Omega)$.

The controlled linearized system around this stationary solution is:

$$
\left\{\begin{array}{c}
y^{\prime}+A_{1} y+P_{\Omega}((\bar{y} \cdot \nabla) y+(y \cdot \nabla) \bar{y}-(\bar{B} \cdot \nabla) B \\
\quad-(B \cdot \nabla) \bar{B})=P_{\Omega}\left(\chi_{\omega} u\right), \\
B^{\prime}+A_{2} B+P_{\Omega}((\bar{y} \cdot \nabla) B+(y \cdot \nabla) \bar{B}-(\bar{B} \cdot \nabla) y \\
\quad-(B \cdot \nabla) \bar{y})=\chi_{\omega} P_{\omega} v
\end{array}\right.
$$

In the linearized system, the second equation contains a supplementary Leray projector necessary in order to keep the solution with divergence free components $y, B$ (see also [3]).

Denote by $\mathcal{A}$ the operator defined by

$$
\mathcal{A}\left(\begin{array}{c}
y \\
B
\end{array}\right)=\left(\begin{array}{l}
A_{1} y+P_{\Omega}((\bar{y} \cdot \nabla) y+(y \cdot \nabla) \bar{y}-(\bar{B} \cdot \nabla) B-(B \cdot \nabla) \bar{B}) \\
A_{2} B+P_{\Omega}((\bar{y} \cdot \nabla) B+(y \cdot \nabla) \bar{B}-(\bar{B} \cdot \nabla) y-(B \cdot \nabla) \bar{y})
\end{array}\right)
$$


with $D(\mathcal{A})=D\left(A_{1}\right) \times D\left(A_{2}\right) \subset H \times H$ and by $\mathcal{B}:\left(L^{2}(\Omega)\right)^{2} \times\left(L^{2}(\Omega)\right)^{2} \rightarrow$ $H \times H$

$$
\mathcal{B}\left(\begin{array}{l}
u \\
v
\end{array}\right)=\left(\begin{array}{c}
P_{\Omega}\left(\chi_{\omega} u\right) \\
\chi_{\omega} P_{\omega} v
\end{array}\right) .
$$

Then, the linear controlled system (5) may be written in abstract form

$$
\left\{\begin{array}{l}
z^{\prime}+\mathcal{A} z=\mathcal{B} w \\
z(0)=z_{0}
\end{array}\right.
$$

where we denoted by $z=(y, B)^{T}, w=(u, v)^{T}$.

As we have mentioned in the Introduction, stabilization of the linear system (6) reduces to the approximate controllability in an arbitrary time $T$ for problem (6) and this is equivalent to the unique continuation property for the dual equation, i.e.

(7) $-\xi^{\prime}(t)+\mathcal{A}^{*} \xi(t)=0$ and $\mathcal{B}^{*} \xi(t)=0, t \in(0, T) \Rightarrow \xi(t)=0, t \in(0, T)$.

To make explicit the dual system, let $\xi=\left(\begin{array}{l}\zeta \\ C\end{array}\right)$ with $\zeta, C$ written as column vectors. Then, the dual of the linearized system is:

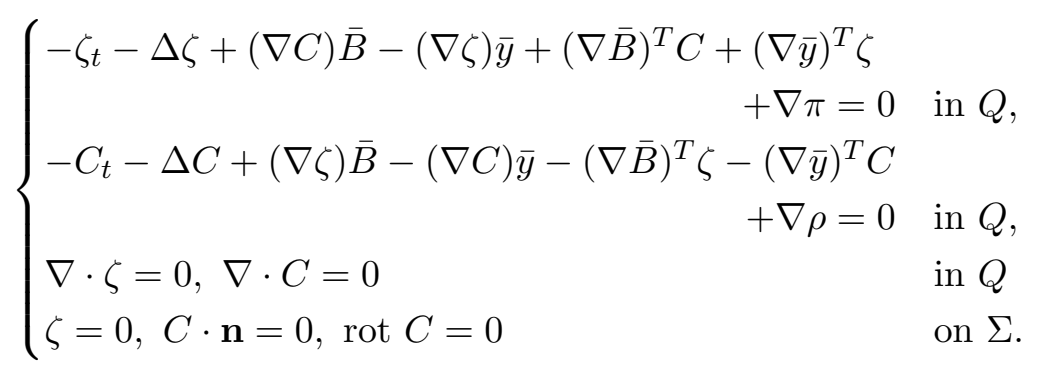

Here, terms like $\nabla C$ or $(\nabla \bar{B})^{T}$ are Jacobian matrices and belong to $\mathcal{M}_{2 \times 2}(\mathbb{R})$, while terms of the type $(\nabla C) \bar{B}$ are simply the matrix products and belong to $\mathcal{M}_{2 \times 1}(\mathbb{R})$. Observe also that $(\nabla C) \bar{B}=(\bar{B} \cdot \nabla) C$.

The adjoint to the control operator is

$$
\mathcal{B}^{*} \xi=\left(\begin{array}{c}
\mathcal{B}_{1}^{*} \zeta \\
\mathcal{B}_{2}^{*} C
\end{array}\right)=\left(\begin{array}{c}
m_{\omega} \zeta \\
P_{\omega} m_{\omega} C
\end{array}\right)
$$

where $m_{\omega}$ denotes the restriction to $\omega$ of functions defined on $\Omega$.

Thus, the main result of this paper, concerning the unique continuation property is: 
Theorem 1. Let $(\zeta, C)^{T}$ be a solution of (8) such that

$$
\zeta \equiv 0, \operatorname{rot} C \equiv 0 \text { in } \omega \times(0, T) \text {. }
$$

Then $\zeta \equiv 0, C \equiv 0$ in $\Omega \times(0, T)$.

3. Proof of Theorem 1. The idea of the proof is to obtain Carleman type estimates for the system (8), in the spirit of the corresponding inequalities used in controllability problems for parabolic and Navier-Stokes equations $([7],[1],[9],[8])$. Since the system contains the pressure terms, we apply the curl operator to the equations in (8) and deduce Carleman type inequalities for the obtained system. However, since we loose boundary conditions for the velocity field, we need to estimate also the corresponding boundary terms. For this, we follow and adapt some ideas from [13]; in fact, a part of the computations, namely the estimates for the general parabolic equations, are almost identical as in the cited reference but we present them for the sake of completeness. However, we have to mention that there is a fundamental difference between this unique continuation result and the usual results for parabolic equations: in the present situation we make use, in an essential way, of the boundary conditions satisfied by the solution.

Step1. Estimates for general parabolic equations. Consider an elliptic operator of the form

$$
L y=\sum_{i, j=1}^{n} a_{i j} \frac{\partial^{2} y}{\partial x_{i} \partial x_{j}}+\sum_{j=1}^{n} b_{j} \frac{\partial y}{\partial x_{j}}+c y
$$

where

i) $a_{i j} \in C^{1}(\Omega), a_{i j}=a_{j i}$ and define a uniformly positive definite matrix: $\sum_{i, j=1}^{n} a_{i j}(x) \xi^{i} \xi^{j} \geq \beta|\xi|^{2}$ for some positive constant $\beta$ and all $x \in \Omega$.

ii) $b_{i}, c \in L^{\infty}\left(0, T ; L^{\infty}(\Omega)\right)$.

In what follows we obtain Carleman type inequalities which have as consequence the well known fact that if a strong solution $y \in L^{2}\left(0, T ; H_{l o c}^{2}(\Omega)\right)$ satisfies

$$
y_{t}+L y=0 \quad \text { in } \Omega \times(0, T)
$$

and $y \equiv 0$ in $\omega \times(0, T)$, then $y \equiv 0$ in $\Omega \times(0, T)$.

To present computations in a simple way, without losing generality, we consider the simplest case of (backward) heat equation:

$$
\frac{\partial y}{\partial t}+\Delta y=f \quad \text { in } \Omega \times(0, T)
$$


with $f \in L^{2}(\Omega \times(0, T))$ and with no boundary conditions specified.

Remark 1. In the estimates we intend to obtain, the $L^{2}$ norm of the solution and its derivatives on $\Omega$ are controlled by the $L^{2}$ norm of the solution on $\omega$, with some weights depending on parameters at our disposal.

Moreover, we shall make the following two assumptions which, in the concrete situation we treat, are not restrictive:

$$
\begin{gathered}
y \in C\left([0, T], H^{2}(\Omega)\right) \\
m:=\inf _{t \in(0, T)} \int_{\Sigma}|y(x, t)|^{2} d \sigma_{x}>0 .
\end{gathered}
$$

In the computations we make, for a given function $z$ we denote by $z_{t}, z_{, i}, z_{, t i}$ etc. the partial derivatives $\frac{\partial z}{\partial t}, \frac{\partial z}{\partial x_{i}}, \frac{\partial^{2} z}{\partial t \partial x_{i}}$ and we use the convention for summation when indices are repeated.

We choose an auxiliary function $\psi \in C^{2}(\bar{\Omega})$ with the properties:

$$
\left.\psi\right|_{\partial \Omega}=1,\left.\psi\right|_{\Omega} \in\left[1, \frac{3}{2}\right],\{x \mid \nabla \psi(x)=0\} \subset \subset \omega, \frac{\partial \psi}{\partial \mathbf{n}}=-1 \text { on } \partial \Omega .
$$

Such a function exists (see [7]). Let for $\lambda>0$

$$
\alpha(x, t)=\frac{e^{\lambda \psi(x)}-e^{2 \lambda}}{t^{2}(T-t)^{2}}, \varphi(x, t)=\frac{e^{\lambda \psi(x)}}{t^{2}(T-t)^{2}}
$$

and $\bar{\alpha}(t)=\left.\alpha(\cdot, t)\right|_{\partial B_{R}}, \bar{\varphi}(t)=\left.\varphi(\cdot, t)\right|_{\partial B_{R}}$. Denote by $z:=e^{s \alpha} y$ for some $s, \lambda>0$. Using (11), the equations satisfied by $z$ is:

$$
z_{t}+\left[z_{, i i}-2 s \alpha_{, i} z_{, i}+\left(s^{2} \alpha_{, i}^{2}-s \alpha_{, i i}-s \alpha_{t}\right) z\right]=f e^{s \alpha} .
$$

We reorder the terms in equation (15) and, denoting by

$$
\begin{gathered}
X(x, t)=\left[z_{, i i}+\left(s^{2} \alpha_{, i}^{2}+s \alpha_{, i i}-s \alpha_{t}\right) z\right], \\
F(x, t)=-2 s\left(\alpha_{, i} z_{, i}+s \alpha_{, i i} z\right),
\end{gathered}
$$

we rewrite (15) as

$$
z_{t}+X(x, t)+F(x, t)=f e^{s \alpha} .
$$


We multiply (16) with $X(x, t)$, we integrate on $Q$ and, by Cauchy inequality we obtain:

$$
\int_{Q} z_{t} X(x, t) d x d t+\int_{Q} X(x, t) F(x, t) d x d t \leq \frac{1}{2} \int_{Q} f^{2} e^{2 s \alpha} .
$$

We proceed as in [7] (see also [1]) to evaluate the integrals in (17). We put in evidence the dominant terms in $s, \lambda, \varphi$ as well as their signs. We observe then that the dominant term concerning $z$ is $s^{3} \lambda^{4} \varphi^{3} z^{2}$ and the other terms, dominated by it, for $s, \lambda$ big enough, like $s^{3} \lambda^{3} \varphi^{3} z^{2}$, will be generically denoted as l.o.t $\left(z^{2}\right)$. The same happens with the terms containing $|\nabla z|^{2}$, where the dominant term is found to be $s \lambda^{2} \varphi|\nabla z|^{2}$ and the lower order terms are generically denoted by l.o.t. $\left(|\nabla z|^{2}\right)$. When talking about $y$ the dominant term will be $s^{3} \lambda^{4} \varphi^{3} e^{2 s \alpha} y^{2}$ and lower order terms like $s^{2} \lambda^{4} \varphi^{2} e^{2 s \alpha} y^{2}$ are denoted shortly as l.o.t. $\left(y^{2}\right)$. Similar notations will be used for the terms containing $|\nabla y|^{2}$. Finally, the dominant terms, with positive sign, will be found in the left part of the final equality. Without supplementary comment we use in the estimations below the fact that $\left|\alpha_{t}\right|,\left|\varphi_{t}\right|,\left|\alpha_{t t}\right| \leq C \varphi^{2}$, with a constant $C$ independent of $\lambda$.

I. $\int_{Q} z_{t} X(x, t) d x d t$ :

$$
\begin{aligned}
& \int_{Q} z_{t} X(x, t) d x d t=\int_{Q} z_{t} z_{, i i}+\left(\frac{z^{2}}{2}\right)_{t}\left(s^{2} \alpha_{, i}^{2}+s \alpha_{, i i}-s \alpha_{t}\right) d x d t \\
& =\int_{\Sigma} z_{t} z_{, i} \mathbf{n}_{i} d \sigma d t-\int_{Q} z_{, t i} z_{, i}+\frac{z^{2}}{2}\left(s^{2} \alpha_{, i}^{2}+s \alpha_{, i i}-s \alpha_{t}\right)_{t} d x d t \\
& =\int_{\Sigma} z_{t} z_{, i} \mathbf{n}_{i} d \sigma d t+\int_{Q} \text { l.o.t. }\left(z^{2}\right) d x d t .
\end{aligned}
$$

II. $\int_{Q} X(x, t) F(x, t) d x d t$ :

$$
\begin{aligned}
& -2 \int_{Q} z_{, i i} s \alpha_{, j} z_{, j} d x d t \\
& =2 \int_{Q} z_{, i} s \alpha_{, i j} z_{, j}+z_{, i} z_{, i j} s \alpha_{, j} d x d t-2 \int_{\Sigma} z_{, i} \mathbf{n}_{i} s \alpha_{, j} z_{, j} d x d t \\
& =\int_{Q} 2 z_{, i} z_{, j} s \alpha_{, i j}-z_{, i}^{2} s \alpha_{, j j} d x d t+\int_{\Sigma} z_{i}^{2} s \alpha_{, j} \mathbf{n}_{j}-2 z_{, i} \mathbf{n}_{i} s \alpha_{, j} z_{, j} \sigma d t .
\end{aligned}
$$




$$
\begin{aligned}
& -2 \int_{Q} z_{, i i} s \alpha_{, j j} z \\
& =2 \int_{Q} z_{, i} z s \alpha_{, i j j}+z_{, i}^{2} s \alpha_{, j j} d x d t-2 \int_{\Sigma} z_{, i} \mathbf{n}_{i} s \alpha_{, j j} z d \sigma d t .
\end{aligned}
$$

$$
-2 \int_{Q} s^{3} \alpha_{, i}^{2} \alpha_{, j} z z, j=\int_{Q} s\left(\alpha_{, i}^{2} \alpha_{, j}\right)_{, j} z^{2} d x d t-\int_{\Sigma} s^{3} \alpha_{, i}^{2} \alpha_{, j} \mathbf{n}_{j} z^{2} d \sigma d t
$$

The other terms in $\int_{Q} X(x, t) F(x, t) d x d t$ are of lower order in $s, \lambda$ and may be evaluated by using Cauchy's inequality.

First estimates, for all solutions

Putting together (18)-(21) we find a constant $C>0$, not depending on the solution $y$, and $\lambda_{0}>0$ such that if $\lambda>\lambda_{0}$ there exists $s_{0}=s_{0}(\lambda)$, such that for all $s>s_{0}$ the following estimate holds:

$$
\begin{aligned}
& \int_{Q \backslash Q_{\omega}} s^{3} \lambda^{4} \varphi^{3} e^{2 s \alpha} y^{2} d x d t+\int_{Q \backslash Q_{\omega}} s \lambda^{2} \varphi e^{2 s \alpha}|\nabla y|^{2}+\int_{\Sigma} \ldots \\
& \leq C\left[\int_{Q_{\omega}}\left(\left(s^{3} \lambda^{3} \varphi^{3}+s^{2} \lambda^{4} \varphi^{2}\right) y^{2}+s \lambda \varphi|\nabla y|^{2}\right) e^{2 s \alpha} d x d t\right] \\
& +\int_{Q} f^{2} e^{2 s \alpha} d x d t .
\end{aligned}
$$

Estimates for the boundary terms

We estimate now the integrals on $\Sigma_{R}$ denoted in the above formula, for short, $\int_{\Sigma} \cdots$. Remember that $\frac{\partial \psi}{\partial \mathbf{n}} \equiv-1$ on $\partial B_{R}$. We will see, after some computations, that concerning the integrals on $\Sigma_{R}$, the dominant term, as a polynomial in $s, \lambda$, is

$$
s^{3} \lambda^{3} \int_{\Sigma_{R}} \bar{\varphi}^{3} e^{2 s \bar{\alpha}}|y|^{2} d \sigma d t=s^{3} \lambda^{3} \int_{0}^{T} e^{2 s \bar{\alpha}} \bar{\varphi}^{3} \int_{\partial B_{R}} y^{2} d \sigma d t
$$

and this turns out to have the right sign + when in the left hand side of the inequality. The other boundary integrals, involving lower degree of the polynomials in $s, \lambda$ will be generically denoted as l.o.t.

$$
-\int_{\Sigma} s^{3} \alpha_{, i}^{2} \alpha, j \mathbf{n}_{j} z^{2} d x d t=s^{3} \lambda^{3} \int_{\Sigma} \bar{\varphi}^{3} e^{2 s \bar{\alpha}}|y|^{2} d \sigma d t
$$


Since $z=e^{s \alpha} y, z_{, i}=\left(s \alpha, i y+y_{, i}\right) e^{s \alpha}$, we have:

$$
\begin{aligned}
& -2 \int_{\Sigma} z_{, i} \mathbf{n}_{i} s \alpha_{, j} z_{, j} d \sigma d t=-2 \int_{\Sigma}\left(s \alpha_{, i} y+y_{, i}\right) \mathbf{n}_{i} s \alpha_{, j}\left(s \alpha_{, j} y+y_{j}\right) e^{2 s \alpha} d \sigma d t \\
& =-2 \int_{\Sigma}\left(s^{3} \alpha_{, i} \alpha_{, j}^{2} \mathbf{n}_{i}|y|^{2}-s y_{, i} \mathbf{n}_{i} \alpha_{, j} y_{, j}\right. \\
& \left.-s^{2} \alpha_{, i} \mathbf{n}_{i} \alpha_{, j} y y_{, j}-s^{2} y_{, i} \mathbf{n}_{i} \alpha_{, j}^{2} y\right) e^{2 s \alpha} d \sigma d t \\
& =2 s^{3} \lambda^{3} \int_{\Sigma} \bar{\varphi}^{3} e^{2 s \bar{\alpha}}|y|^{2} d \sigma d t+\int_{\Sigma} \text { l.o.t.d } \sigma d t \text {. }
\end{aligned}
$$

$$
\begin{aligned}
\int_{\Sigma} z_{, i}^{2} s \alpha_{, j} \mathbf{n}_{j} d \sigma d t & =\int_{\Sigma}\left(s \alpha_{, i} y+y_{, i}\right)^{2} s \alpha_{, j} \mathbf{n}_{j} e^{2 s \alpha} d \sigma d t \\
& =\int_{\Sigma} s^{3} \alpha_{, i}^{2} \alpha_{, j} \mathbf{n}_{j} e^{2 s \alpha} y^{2}+y_{, i}^{2} s \alpha_{, j} \mathbf{n}_{j} e^{2 s \alpha} \\
& +2 s \alpha_{, i} y y_{, i} s \alpha_{, j} \mathbf{n}_{j} e^{2 s \alpha} d \sigma d t \\
& =-s^{3} \lambda^{3} \int_{\Sigma} \bar{\varphi}^{3} e^{2 s \bar{\alpha}} y^{2} d \sigma d t+\int_{\Sigma} \text { l.o.t.d } \sigma d t .
\end{aligned}
$$

$$
-2 \int_{\Sigma} z_{, i} \mathbf{n}_{i} s \alpha_{, j j} z d \sigma d t=-2 \int_{\Sigma}\left(s \alpha_{, i} y+y_{i}\right) \mathbf{n}_{i} s \alpha_{, j j} y e^{2 s \alpha} d \sigma d t
$$

$$
=-2 \int_{\Sigma} s^{2} \alpha_{, i} \mathbf{n}_{i} \alpha, j j y^{2} e^{2 s \alpha} d \sigma d t-2 \int_{\Sigma} s \alpha, j j \frac{\partial y}{\partial \mathbf{n}} y e^{2 s \alpha} d \sigma d t
$$

$$
\begin{aligned}
\int_{\Sigma} z_{t} z_{, i} \mathbf{n}_{i} d \sigma d t & =\int_{\Sigma}\left(s \alpha_{t} y+y_{t}\right)\left(s \alpha_{, i} y+y_{, i}\right) \mathbf{n}_{i} e^{2 s \alpha} d \sigma d t \\
& =s^{2} \int_{\Sigma}\left(\alpha_{t} \alpha_{, i} \mathbf{n}_{i} y^{2}+\text { l.o.t. }\right) e^{2 s \alpha} d \sigma d t .
\end{aligned}
$$

Final estimates for the given solution $y$

If we look now to the formulas (23)-(27), after summation, we find that the dominant term for the integral on $\Sigma$, is

$$
\begin{aligned}
& 2 s^{3} \lambda^{3} \int_{\Sigma} \varphi^{3} y^{2} e^{2 s \alpha} d \sigma d t \\
& =2 s^{3} \lambda^{3} \int_{0}^{T} e^{2 s \bar{\alpha}} \bar{\varphi}^{3} \int_{\partial \Omega} y^{2} d \sigma d t \geq 2 m s^{3} \lambda^{3} \int_{0}^{T} e^{2 s \bar{\alpha}} \bar{\varphi}^{3} d t .
\end{aligned}
$$


The dominant term on the boundary has positive sign and belongs to the left side of the inequality. In this situation, for $s, \lambda>0$ big enough, the sum of the integrals on $\Sigma$ becomes positive, that is the lower order terms are absorbed by the dominant term.

So, we find that there exist a constant $C=C(y)>0$ and $\lambda_{0}>0$ such that if $\lambda>\lambda_{0}$ there exists $s_{0}=s_{0}(\lambda)$, such that for all $s>s_{0}$ the following final estimate holds:

$$
\begin{aligned}
& \int_{Q \backslash Q_{\omega}} s^{3} \lambda^{4} \varphi^{3} e^{2 s \alpha}|y|^{2}+s \lambda^{2} \varphi e^{2 s \alpha}|\nabla y|^{2} d x d t+\int_{\Sigma} s^{3} \lambda^{3}|y|^{2} e^{2 s \bar{\alpha}} \bar{\varphi}^{3} d \sigma d t \\
& \leq C\left(\int_{Q_{\omega}} s^{3} \lambda^{4} \varphi^{3} e^{2 s \alpha}|y|^{2}+s \lambda^{2} \varphi e^{2 s \alpha}|\nabla y|^{2} d x d t\right)+\int_{Q} f^{2} e^{2 s \alpha} d x d t
\end{aligned}
$$

This implies immediately that if $f \equiv 0$ and $y=0$ on $\omega \times(0, T)$ then necessarily $y=0$ on $\Omega \times(0, T)$.

Step 2. Unique continuation for system (8). We apply the rot operator to the equations in (8). We denote by $v=\operatorname{rot} \zeta, w=\operatorname{rot} C$. Observe that $w=0$ on $\Sigma$ but no boundary conditions are known for $v$. We obtain the following system in $v, w$ :

$$
\begin{cases}-v_{t}-\Delta v-(\bar{y} \cdot \nabla) v+(\bar{B} \cdot \nabla) w=-2 \operatorname{rot}\left((\nabla \bar{y})^{T} \zeta\right) & \text { in } Q \\ -w_{t}-\Delta w+(\bar{B} \cdot \nabla) v-(\bar{y} \cdot \nabla) w=2 \operatorname{rot}\left((\nabla \bar{B})^{T} \zeta\right) & \text { in } Q\end{cases}
$$

The inequality (28) adapted to system (29), gives the following estimate

$$
\begin{aligned}
& \int_{Q \backslash Q_{\omega}} s^{3} \lambda^{4} \varphi^{3} e^{2 s \alpha}\left(|v|^{2}+|w|^{2}\right)+s \lambda^{2} \varphi e^{2 s \alpha}\left(|\nabla v|^{2}+|\nabla w|^{2}\right) d x d t \\
(30) & +\int_{\Sigma} s^{3} \lambda^{3} e^{2 s \bar{\alpha}} \bar{\varphi}^{3}|v|^{2} d \sigma d t \\
& \leq C\left(\int_{Q_{\omega}} s^{3} \lambda^{4} \varphi^{3} e^{2 s \alpha}\left(|v|^{2}+|w|^{2}\right)+s \lambda^{2} \varphi e^{2 s \alpha}\left(|\nabla v|^{2}+|\nabla w|^{2}\right) d x d t\right) \\
& +C \int_{Q}\left(|\zeta|^{2}+|\nabla \zeta|^{2}\right) e^{2 s \alpha} d x d t .
\end{aligned}
$$

Denote by $\theta$ the stream function of $\zeta: \zeta=\left(-\theta_{, 2}, \theta_{, 1}\right)^{T}$. Since $\zeta=0$ on the boundary we have that $\nabla \theta=0$ on $\partial \Omega$ and by the fact that the domain is simply connected we may choose $\theta=0$ on $\partial \Omega$. Observe that

$$
\Delta \theta=v \text { in } \Omega .
$$


We need the following theorem of IMANUVILOV and PUEL established in [11], [10]:

Theorem 2. Let $\Omega \subset \mathbb{R}^{n}$ be bounded, open, with $C^{2}$ boundary. Let $y \in H^{1}(\Omega)$ be solution of the following boundary value problem:

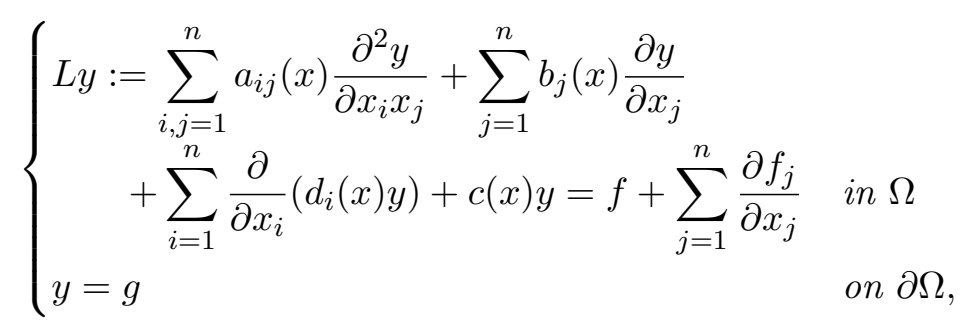

where

i) $a_{i j} \in C^{2}(\bar{\Omega}), b_{j}, c, d_{i} \in L^{\infty}(\Omega), i, j=\overline{1, n}, a_{i j}$ verify the uniform ellipticity condition

$$
\sum_{i, j=1}^{n} a_{i j}(x) \xi^{i} \xi^{j} \geq \beta|\xi|^{2}, \forall \xi \in \mathbb{R}^{n}, x \in \Omega
$$

for some $\beta>0$.

ii) $f, f_{j} \in L^{2}(\Omega), j=\overline{1, n}, g \in H^{\frac{1}{2}}(\partial \Omega)$.

Consider a function $\psi \in C^{2}(\bar{\Omega})$ with the following properties, analogous to (14):

$$
\left.\psi\right|_{\partial \Omega}=0,\left.\psi\right|_{\Omega}>0,\{x \mid \nabla \psi(x)=0\} \subset \omega \subset \subset \Omega, \frac{\partial \psi}{\partial \mathbf{n}}<0 \text { on } \partial \Omega .
$$

With $\eta(x)=e^{\lambda \psi(x)}$, there exist $\hat{\lambda}, \hat{\tau}$ and a constant $C>0$ such that for all $\lambda \geq \hat{\lambda}, \tau \geq \hat{\tau}$ the following inequality holds:

$$
\begin{aligned}
& \int_{\Omega} e^{2 \tau \eta}|\nabla y|^{2} d x+\tau^{2} \lambda^{2} \int_{\Omega} \eta^{2} e^{2 \tau \eta}|y|^{2} d x \\
& \leq C\left[\tau^{\frac{1}{2}} e^{2 \tau}\|g\|_{H^{\frac{1}{2}(\partial \Omega)}}^{2}+\frac{1}{\tau \lambda^{2}} \int_{\Omega} \frac{|f|^{2}}{\eta} e^{2 \tau \eta} d x\right. \\
& \left.+\sum_{j=1}^{n} \tau \int_{\Omega}\left|f_{j}\right|^{2} \eta e^{2 \tau \eta} d x+\int_{\omega}\left(|\nabla y|^{2}+\tau^{2} \lambda^{2} \eta^{2}|y|^{2}\right) e^{2 \tau \eta} d x\right] .
\end{aligned}
$$

Observe that when $g=0$ we may choose $\psi$ as in (14) and keep estimate (33) valid. 
We rewrite now (31) as

$$
\begin{cases}\Delta \nabla \theta=\nabla v & \text { in } \Omega \\ \nabla \theta=0 & \text { on } \partial \Omega .\end{cases}
$$

We apply now inequality (33) to $\nabla \theta$ for fixed $t$ and we obtain, for all $\tau>\widehat{\tau}, \lambda>\widehat{\lambda}$, the inequality

$$
\begin{aligned}
& \int_{\Omega} e^{2 \tau \eta}\left(\left|D^{2} \theta\right|^{2}+\tau^{2} \lambda^{2} \eta^{2}|\nabla \theta|^{2}\right) d x \\
& \leq C\left[\tau \int_{\Omega}|v|^{2} \eta e^{2 \tau \eta} d x+\int_{\omega}\left(\left|D^{2} \theta\right|^{2}+\tau^{2} \lambda^{2} \eta^{2}|\nabla \theta|^{2}\right) e^{2 \tau \eta} d x\right] .
\end{aligned}
$$

We choose $\tau=\frac{s}{t^{2}(T-t)^{2}}$ in this inequality, then we multiply (35) by $e^{-\frac{2 s}{t^{2}(T-t)^{2}} e^{2 \lambda\|\psi\|} C(\bar{\Omega})}$ and integrate from 0 to $T$. We obtain:

$$
\begin{gathered}
\int_{Q} e^{2 s \alpha}\left(\left|D^{2} \theta\right|^{2}+s^{2} \lambda^{2} \varphi^{2}|\nabla \theta|^{2}\right) d x d t \\
\leq C\left[\int_{Q}|v|^{2} s \varphi e^{2 s \alpha} d x d t+\int_{Q_{\omega}} e^{2 s \alpha}\left(\left|D^{2} \theta\right|^{2}+s^{2} \lambda^{2} \varphi^{2}|\nabla \theta|^{2}\right) d x d t\right]
\end{gathered}
$$

and, considering that $\theta$ is the stream function of $\zeta$ this implies that

$$
\begin{aligned}
& \int_{Q} e^{2 s \alpha}\left(|\nabla \zeta|^{2}+s^{2} \lambda^{2} \varphi^{2}|\zeta|^{2}\right) d x d t \\
& \leq C\left[\int_{Q}|v|^{2} s \varphi e^{2 s \alpha} d x d t+\int_{Q_{\omega}} e^{2 s \alpha}\left(|\nabla \zeta|^{2}+s^{2} \lambda^{2} \varphi^{2}|\zeta|^{2}\right) d x d t\right] .
\end{aligned}
$$

We plug now inequality (37) in the right hand side of (30) and obtain, after reducing the lower order terms concerning $v$ the inequality

$$
\begin{aligned}
& \int_{Q \backslash Q_{\omega}} s^{3} \lambda^{4} \varphi^{3} e^{2 s \alpha}\left(|v|^{2}+|w|^{2}\right)+s \lambda^{2} \varphi e^{2 s \alpha}\left(|\nabla v|^{2}+|\nabla w|^{2}\right) d x d t \\
(38) & +\int_{\Sigma} s^{3} \lambda^{3} e^{2 s \bar{\alpha}} \bar{\varphi}^{3}|v|^{2} d \sigma d t \\
& \leq C\left(\int_{Q_{\omega}} s^{3} \lambda^{4} \varphi^{3} e^{2 s \alpha}\left(|v|^{2}+|w|^{2}\right)+s \lambda^{2} \varphi e^{2 s \alpha}\left(|\nabla v|^{2}+|\nabla w|^{2}\right) d x d t\right) \\
& +C \int_{Q_{\omega}} e^{2 s \alpha}\left(|\nabla \zeta|^{2}+s^{2} \lambda^{2} \varphi^{2}|\zeta|^{2}\right) d x d t .
\end{aligned}
$$


This immediately implies that, if $\zeta=0, w=\operatorname{rot} C=0$ in $Q_{\omega}$ then $v=\operatorname{rot} \zeta \equiv 0, w=\operatorname{rot} C \equiv 0$ in $Q$. But, since the domain is simply connected, it follows that $\zeta \equiv 0, C \equiv 0$ in $Q$.

\section{REFERENCES}

1. BARBU, V. - Controllability of parabolic and Navier-Stokes equations, Sci. Math. Jpn., 56 (2002), 143-211.

2. BARBU, V. - Feedback stabilization of Navier-Stokes equations, ESAIM Control Optim. Calc. Var., 9 (2003), 197-206.

3. Barbu, V.; Havârneanu, T.; Popa, C.; Sritharan, S.S. - Local exact controllability for the magnetohydrodynamic equations revisited, Adv. Differential Equations, 10 (2005), 481-504.

4. BARBU, V.; LASIECKA, I.; Triggiani, R. - Abstract settings for tangential boundary stabilization of Navier-Stokes equations by high- and low-gain feedback controllers, Nonlinear Anal., 64 (2006), 2704-2746.

5. Barbu, V.; Lasiecka, I.; Triggiani, R. - Tangential Boundary Stabilization of Navier-Stokes Equations, Mem. Amer. Math. Soc. 181, 2006.

6. Barbu, V.; Triggiani, R. - Internal stabilization of Navier-Stokes equations with finite-dimensional controllers, Indiana Univ. Math. J., 53 (2004), 1443-1494.

7. Fursikov, A.V.; Imanuvilov, O. Yu. - Controllability of Evolution Equations, volume 34 of Lecture Notes Series, Seoul National University Research Institute of Mathematics Global Analysis Research Center, Seoul, 1996.

8. Imanuvilov, O. Yu. - On exact controllability for the Navier-Stokes equations, ESAIM Control Optim. Calc. Var., 3 (1998), 97-131.

9. Imanuvilov, O. Yu. - Remarks on exact controllability for the Navier-Stokes equations, ESAIM Control Optim. Calc. Var., 6 (2001), 39-72.

10. Imanuvilov, O. Yu.; Puel, J.-P. - Global Carleman estimates for weak solutions of elliptic nonhomogeneous Dirichlet problems, C. R. Math. Acad. Sci. Paris, 335 (2002), 33-38.

11. Imanuvilov, O. Yu.; Puel, J.-P. - Global Carleman estimates for weak solutions of elliptic nonhomogeneous Dirichlet problems, Int. Math. Res. Not., 16 (2003), 883-913.

12. Ladyženskaja, O. A.; Solonnikov, V. A. - The linearization principle and invariant manifolds for problems of magnetohydrodynamics, (Russian) Boundary value problems of mathematical physics and related questions in the theory of functions, 7 . Zap. Naucn. Sem. Leningrad. Otdel. Mat. Inst. Steklov. (LOMI), 38 (1973), 46-93.

13. LeFter, C. - Feedback stabilization of magnetohydrodynamic equations, to appear, Preprint nr. 1144/2006 - WIAS - Berlin. 
14. LeFter, C. - Feedback stabilization of two dimensional magnetohydrodynamic equations, An. Ştiinţ. Univ. "Al.I. Cuza" Iaşi, Ser. Noua, Mat., 55 (2009), 21-34.

15. SRITHARAN, S.S. - Invariant Manifold Theory for Hydrodynamic Transition, Pitman Research Notes in Mathematics Series, 241, Longman Scientific \& Technical, Harlow; copublished in the United States with John Wiley \& Sons, Inc., New York, 1990.

16. Yudovich, V.I. - The Linearization Method in Hydrodynamical Stability Theory, Translations of Mathematical Monographs, 74, American Mathematical Society, Providence, RI, 1989.

Received: 15.XII.2008

Faculty of Mathematics, University "Al.I. Cuza",

11, Bd. Carol I, 700506, Iaşi, ROMANIA

and

Institute of Mathematics "Octav Mayer",

Romanian Academy, Iaşi Branch, ROMANIA

catalin.lefter@uaic.ro 
